\title{
Realizing Planar Graphs as Convex Polytopes
}

\author{
Günter Rote \\ Institut für Informatik, Freie Universität Berlin, \\ Takustraße 9, 14195 Berlin, Germany \\ rote@inf.fu-berlin.de
}

\begin{abstract}
This is a survey on methods to construct a three-dimensional convex polytope with a given combinatorial structure, that is, with the edges forming a given 3-connected planar graph, focusing on efforts to achieve small integer coordinates.
\end{abstract}

Keywords: Convex polytope, spiderweb embedding.

\section{Introduction}

The graphs formed by the edges of three-dimensional polytopes are characterized by Steinitz' seminal theorem from 1916 [13]: they are exactly the planar 3connected graphs. For such a graph $G$ with $n$ vertices, I will discuss different methods of actually constructing a polytope with this structure.

\section{Inductive Methods}

The original proof of Steinitz transforms $G$ into simpler and simpler graphs by sequence of elementary operations, until eventually $K_{4}$, the graph of the tetrahedron, is obtained. By following this transformation in the reverse order, one can gradually turn the tetrahedron into a realization of $G$. The operations can be carried out with rational coordinates, and after clearing common denominators, one obtains integer coordinates. However, the required number of bits of accuracy for each vertex coordinate is exponential. In other words, the $n$ vertices lie on an integer grid whose size is doubly exponential in $n$ [9].

A triangulated (or simplicial) polytope, in which every face is a triangle, is easier to realize on the grid than a general polytope, since each vertex can be perturbed within some small neighborhood while maintaining the combinatorial structure of the polytope.

Das and Goodrich 5] showed that triangulated polytopes can be embedded with coordinates of size $O\left(2^{\text {poly }(n)}\right)$, by performing $O(\log n)$ stages of many independent Steinitz operations in parallel. (An explicit bound on the coordinates has not been worked out for this method.) 


\section{$3 \quad$ Tutte Embeddings}

The Schlegel diagram of a polytope $P$ is obtained by a central projection from a point $O$ that is outside $P$ but sufficiently close to a face $F$ of $P$ such that $F$ is the only face that $F$ sees. In the Schlegel diagram, $F$ will appear as the outer face, and the remaining faces will tile $F$ without overlap. Thus, the Schlegel diagram is a plane drawing of the graph $G$ with convex faces (including the outer face).

There are a number of methods that first construct such a plane drawing of $G$ and then lift it to three dimensions. Convex faces are by no means sufficient to guarantee that a drawing is a Schlegel diagram. A characterization of Schlegel diagrams is provided by the so-called Maxwell-Cremona correspondence, observed by Maxwell in 1864 [8], which is described below. By a projective transformation, we can assume that the graph $G$ is drawn in the $x y$-plane, and the projection center $O$ is at infinity at the positive $z$-axis. In other words, the projection is vertical and consists in projecting away the $z$-coordinate.

An equilibrium stress assigns a force to every edge such that in every vertex, the forces cancel. The forces on an edge pull ("positive stress") or push ("negative stress") on both endpoints with the same magnitude, in the direction parallel to the edge.

Theorem 1 (Maxwell, Whiteley [18]). Let $G$ be a planar 3-connected graph drawn in the plane without crossings. The following are equivalent:

- $G$ is the vertical projection of a convex polytope.

- There is an equilibrium stress on $G$ which is positive on the interior edges and negative on the boundary edges.

This theorem is constructive, in the sense that the lifting can be computed in a straightforward way from the equilibrium stress, and vice versa.

To construct a plane embedding that has an equilibrium stress, one can use the spider-web approach suggested by Tutte [15 16]: after fixing the positions of the vertices of the outer face in the shape of a convex polygon, we stipulate that the forces on the interior edges should be not just parallel to, but equal to the edge vectors. The equilibrium condition amounts now to requiring that every interior vertex should lie at the barycenter of its neighbors. This leads to a linear system of equations for the positions of the vertices. After solving this system, there is equilibrium at the interior vertices. However, equilibrium at the boundary vertices is only guaranteed when the outer face is a triangle. If this is not the case, one can realize the polar polytope $P^{*}$, whose graph $G^{*}$ is the dual of $G$, instead: either $G$ or $G^{*}$ must contain a triangle. The calculations for the polarization operation increase the size of the coordinates, leading to bounds of $O\left(\right.$ const $\left.^{n^{2}}\right)$ [11. A linear exponent of $O\left(188^{n}\right)$ has finally been achieved by Rote, Ribó and Schulz [10]: if the outer face is a quadrilateral or a pentagon, one can choose its shape in an appropriate way, in order to ensure that equilibrium also holds on the boundary, and polarization is not needed. This last paper establishes a connection between the size of the coordinates and the number of spanning trees of $G$. Due to improved upper bounds on the number of spanning trees of a planar graph [4, the best bound on the coordinates is currently $O\left(147.71^{n}\right)$. 


\subsection{Stacked Polytopes}

A stacked polytope is obtained by starting with a tetrahedron and repeatedly gluing a new tetrahedron onto some face. Its graph is a 3-tree: It is obtained from $K_{4}$ by repeatedly drawing a new vertex into a triangular face and connecting it to the three triangle vertices.

In a recent first breakthrough on the way towards providing polynomial grid embeddings for polytopes, Demaine and Schulz [6] (after some more specialized cases treated by Zickfeld [19]) showed that every stacked polytope with $n$ vertices can be realized on a polynomial grid of size $O\left(n^{4}\right) \times O\left(n^{4}\right) \times O\left(n^{18}\right)$.

Stacked polytopes are a special class of triangulated polytopes, and, due to their hierarchical structure, they are somewhat easier to handle. Sill, they are sufficiently varied so that one might hope to extend the techniques to, say, all triangulated polytopes.

\section{Nonlinear Methods}

For completeness, I will mention some other construction methods for polytopes, which, however, don't lend themselves to achieving integer realizations.

Midscribed Polytopes. An alternative proof of Steinitz' theorem applies the Koebe-Andreyev-Thurston Circle Packing Theorem (see for example [12]). This theorem can be used to produce a polytope whose edges are tangent to a sphere, that is, they are mid-scribed around the sphere (instead of circumscribed or inscribed). One can define a converging process that yields such a polytope. However, the exact mid-scribed realization (which is unique up to Möbius transformations) necessarily boils down to a nonlinear system of equations, and there are polytopes for which such a realization must have irrational coordinates. It is conceivable that an "approximately mid-scribed" polytope might be good enough, at least for triangulated graphs, but this has not been investigated.

The Colin de Verdière number. Lovász 7 showed that an $n \times n$ matrix of rank 3 that arises in the definition of the Colin de Verdière parameter $\mu(G)$ of a graph $G$ (which equals 3 for graphs of polytopes), can be used to construct coordinates for a polytope realization. However, it is not easy to find this matrix.

\section{Lower Bounds}

The known lower bounds on a grid embedding of a 3-polytope as disappointingly weak. A convex $n$-gon with integral vertices needs an area of $\Omega\left(n^{3}\right)$ in the plane 12 14117. Therefore, realizing a 3-polytope with an $(n-1)$-gonal face requires at least one dimension of size $\Omega\left(n^{3 / 2}\right)$. Given that only an exponential upper bound is known, this is very weak. If one is just interested in strictly convex faces, then a drawing on an $O\left(n^{2}\right) \times O\left(n^{2}\right)$ grid is possible 3. The true bound is not known, but in this case the gap to the lower bound $\Omega\left(n^{3 / 2}\right) \times \Omega\left(n^{3 / 2}\right)$ is not so big. 


\section{References}

1. Acketa, D.M., Žunić, J.D.: On the maximal number of edges of convex digital polygons included into an $m \times m$-grid. J. Comb. Theory Ser. A 69(2), 358-368 (1995)

2. Andrews, G.E.: A lower bound for the volume of strictly convex bodies with many boundary lattice points. Trans. Amer. Math. Soc. 99, 272-277 (1961)

3. Bárány, I., Rote, G.: Strictly convex drawings of planar graphs. Documenta Math. 11, 369-391 (2006)

4. Buchin, K., Schulz, A.: On the Number of Spanning Trees a Planar Graph can have. In: de Berg, M., Meyer, U. (eds.) ESA 2010. LNCS, vol. 6346, pp. 110-121. Springer, Heidelberg (2010)

5. Das, G., Goodrich, M.T.: On the complexity of optimization problems for 3 -dimensional convex polyhedra and decision trees. Comput. Geom. Theory Appl. 8(3), 123-137 (1997)

6. Demaine, E.D., Schulz, A.: Embedding stacked polytopes on a polynomial-size grid. In: Proceedings of the 22nd Annual ACM-SIAM Symposium on Discrete Algorithms (SODA), San Francisco, pp. 1177-1187 (2011)

7. Lovász, L.: Steinitz representations of polyhedra and the Colin de Verdière number. J. Comb. Theory, Ser. B 82, 223-236 (2000)

8. Maxwell, J.C.: On reciprocal figures and diagrams of forces. Phil. Mag. Ser. 27, 250-261 (1864)

9. Onn, S., Sturmfels, B.: A quantitative Steinitz' theorem. In: Beiträge zur Algebra und Geometrie, vol. 35, pp. 125-129 (1994)

10. Ribó Mor, A., Rote, G., Schulz, A.: Small grid embeddings of 3-polytopes. Discrete and Computational Geometry 45, 65-87 (2011),

http://page.mi.fuberlin.de/rote/Papers/pdf/Small+grid+embeddings+of+3polytopes.pdf

11. Richter-Gebert, J.: Realization Spaces of Polytopes. Lecture Notes in Mathematics, vol. 1643. Springer, Heidelberg (1996)

12. Schramm, O.: Existence and uniqueness of packings with specified combinatorics. Israel J. Math. 73, 321-341 (1991)

13. Steinitz, E.: Polyeder und Raumeinteilungen. In: Encyclopädie der mathematischen Wissenschaften, vol. III.1.2 (Geometrie), chap. IIIAB12, pp. 1-139. B. G. Teubner, Leipzig (1922)

14. Thiele, T.: Extremalprobleme für Punktmengen. Master's thesis, Freie Universität Berlin (1991)

15. Tutte, W.T.: Convex representations of graphs. Proceedings London Mathematical Society 10(38), 304-320 (1960)

16. Tutte, W.T.: How to draw a graph. Proceedings London Mathematical Society $13(52), 743-768$ (1963)

17. Voss, K., Klette, R.: On the maximal number of edges of convex digital polygons included into a square. Počítače a umelá inteligencia 1(6), 549-558 (1982) (in Russian)

18. Whiteley, W.: Motion and stresses of projected polyhedra. Structural Topology 7 , 13-38 (1982)

19. Zickfeld, F.: Geometric and Combinatorial Structures on Graphs. Ph.D. thesis, Technical University Berlin (December 2007) 